\title{
Implant Bed Preparation for Immediate Implantation in Molar Region: An Alternative Approach.
}

\author{
Dr .Ekta Rohra ${ }^{1}$,Dr. Gaurang Mistry ${ }^{2}$,Dr. Tej Joshi ${ }^{3}$, \\ Dr. Unmesh Khanvilkar ${ }^{4}$
}

\begin{abstract}
The concept of immediate implant placement has become populardue to less trauma, reduced overall treatment time, decreased patient's anxiety and discomfort, high patient acceptance and better function, and esthetics.Removal of molar teeth provides a challenging and intriguing dilemma due to multiple root morphology. In cases of extraction and immediate placement of dental implants in the molar region preserving alveolar bone is necessary in aiding in osseointergration. Minimal alveolar bone removal should be considered and attained to provide an acceptable surgical site for successful placement of the dental implant. Perhaps most importantly when considering immediate molar implant placement, removal of the intra-radicular septum should be avoided to allow the attainment of initial implant stability at the time of placement and prevent the slipping of the implant into the extraction socket. Thus following this technique will prevent the slip of the implant and allow the implant to be guided into the inter-radicular septum to attain maximum primary stability.
\end{abstract}

Keywords: Guided surgery, immediate placement, implants bed preparation.

\section{Introduction}

The reports of placing implants in fresh extraction sockets were by Schulte and Heimke and Schulte et al, who described this procedure as "immediate implant placement." Immediate implant placement, defined as the placement of dental implant immediately into fresh extraction socket site after tooth extraction, has been considered a predictable and acceptable procedure. Becker et al reported a 93.3\% 5-year implant survival rate with clinically insignificant crestal alveolar bone loss for immediate implants that were augmented with barrier membranes. ${ }^{3-5}$

Original protocols required the placement of implants into healed edentulous ridges and implant placement signifies the placement of the implant in the healed extracted socket after a minimum of 5-6 months. In 1989, Lazzara $^{7}$ placed implants at the time of tooth extraction. Over the past few years, numerous studies have confirmed the reliability of implants placed at the time of tooth extraction. The natural socket being rich in periodontal cells and matrix makes the healing faster and more predictable.

Immediate implants achieve survival rates comparable to those of delayed implants placed in healed sites, ${ }_{8-11}$ particularly for single-tooth replacement in the esthetic zone but also in molar regions. To achieve excellence when placing immediate implants, the diagnosis and planning of the case must be precise. The patient should not have any contraindications to treatment, such as systemic diseases, and he should not be consuming any prescription medications or recreational drugs, the buccal and lingual plate of the extraction socket must be present, the teeth adjacent to the extraction socket must be free of overhanging or insufficient restoration margins, the patient most preferably should not use nicotine; and the interradicular septum should be wide and intact following the tooth extraction. ${ }^{6}$

To predict good prognosis in patients also the placement of the implant plays an important role, over the years the use of auto polymerized resin, vaccum formed template, gutta purcha guided template, metal sleeves, etc, have all been used as surgical guides. In this case we use the tooth itself as a surgical guided to prepare the implant bed as well as to place the implant into the prepared implant bed space.

\section{Case report}

A male patient of the age of 26 had come to the department of prosthodontics, with a complaint of a mutilated tooth in the left mandibular region (Fig.1). On radiographic examination it was concluded that the patient had root caries and was advised extraction of \#36. Following which the treatment options were given to the patient. Options involved extraction of tooth and augmentation of the socket, followed by a delayed implant placement. Option involved extraction of tooth and fabrication of a metal-ceramic bridge using adjacent teeth as abutments. The fourth option was immediate implant placement. The patient agreed to the option of having an immediate implant 
placement. The patient was informed about the treatment procedures 1 week prior to the surgery, the information was provided and the consent form was signed. The patient fulfilled the following required criteria before undergoing treatment, the patient had no contraindications to treatment, such as systemic diseases (eg, diabetes), and he was not consuming any prescription medications or recreational drugs, the buccal and lingual plate of the extraction socket was present, the teeth adjacent to the extraction socket were free of overhanging or insufficient restoration margins, the patient did not use nicotine; and the inter-radicular septum was wide. It was decided to hence carry out a guided surgery for placement of the implant, the guided used was the tooth itself.

Pre surgical preparations were done; an inferior alveolar nerve block was administered in the third quadrant, the tooth was sectioned into a mesial and distal sections (Fig.2). Both sections were luxated slightly using an elevator (Fig.3). Following which the tooth was not extracted, instead the nonextracted roots were considered as surgical guided for the placement of the implant at the furcation. Osteotomy was performed to place a tapered implant of $4.65 \mathrm{~mm} \times 11.5 \mathrm{~mm}$ (AlphaBio Ice). Implant was placed within the prepared socket with the roots still intact within the socket, to avoid the implant from slipping into the extraction socket (Fig.4and 5). A primary stability of $15 \mathrm{Nm}$ was achieved. Once the implant was placed the tooth roots were then extracted atraumatically and checked for any remaining root piece with an X-ray (Fig6 and 7). The sockets were filled with regenerative bone matrix (Ossifi). The site was sutured and allowed to heal for 3 months (Fig.8 and 9) After complete healing the second stage surgery was performed, followed by impression made by closed tray technique. A ceramo-metal crown was cemented. (Fig.8 to 12)

\section{Discussion}

Immediate treatment approaches are gaining popularity in implant dentistry. With careful patient selection and accurate clinical procedures, immediate implants perform predictably well and achieve survival rates that are comparable to those of delayed implants placed in healed sites. The case presentations in this article demonstrate an approach that allowed for improved guidance during implant bed preparation for immediate implants at multi-rooted molar sites. With the osteotomy drills stabilized and guided by the retained root aspects, this new technique allows for precise positioning and angulation of the implant bed preparation in the presence of any inter-radicular bone septa at multirooted extraction sockets. Pre-extractive inter-radicular implant bed preparation may be regarded as a useful tool to in immediate implant placement. Moreover, it should be noted that when this technique is applied, the initially retained root complex serves as an ideal template for the emergence profile of the tooth to be replaced. ${ }^{15}$

Drilling through the dentin of retained root aspects appears to be similar to drilling through tissue slightly harder than dense cortical bone but ultimately is without any specific difficulty. This supports the results of Davarpanah and Szmukler-Moncler, who reported on implant placement in contact with ankylosed root fragments. In this context, it is recommended to use new drills when preextractive inter-radicular implant bed preparation is to be employed. ${ }^{14}$ As a consequence, immediate placement of implants into fresh extraction sockets consistently results in a certain peri-implant marginal defect between the implant and walls of the socket. Therefore, in the literature, a variety of clinical approaches have been advocated to combine immediate implant placement with different regenerative procedures. ${ }^{15}$

\section{Conclusion}

This novel form of implant bed preparation may be regarded as an uncomplicated but useful modification of the standard procedure that allows for ideal implant positioning during immediate implant placement at multi-rooted extraction sites. Even though several studies prove the advantages of immediate implant placement over delayed implant placement case selection, proper diagnosis, treatment planning, and initial stability are very important factors for the success of an immediate implant. Several studies even show that there is not much of marginal bone loss difference between immediate and delayed implant placement. Nevertheless, beyond doubt, immediate implant placement saves time and needs less invasive surgical procedures with considerably very good esthetic outcome when final restorations are placed. Author's personal opinion is immediate implant placement in the extracted tooth socket is a well-accepted and practical treatment worldwide which gives excellent success rates.

\section{Clinical Significance}

The extraction of the roots was done after placement of the implant, as it acts a guide from proper positioning of the implant and prevents the implant from slipping into the extraction socket. 
Care should be taken while extraction of the root pieces once the implant is placed as it can hamper the primary stability.

\section{Reference}

[1]. Schulte W, Heimke G. The Tübinger immediate implant. Quintessenz 1976;27:17-23.

[2]. Schulte W, Kleineikenscheidt H, Lindner K, Schareyka R. The Tübingen immediate implant in clinical studies [in German]. Dtsch Zahnarztl Z 1978;33:348-359.

[3]. Becker W, Becker BE, Polizzi G, Bergstrom C. Autogenous bone grafting of bone defects adjacent to implants placed into immediate extraction sockets in patient: a prospective study. Int J Oral Maxillofac Implants 1994: 9: 389-396.

[4]. Becker W, Dahlin C, Becker BE, Lekholm U, van Steenberghe D, Higuchi K, Kultje C. The use of e-PTFE barrier membranes for bone promotion around titanium implants placed into extraction sockets: a prospective multicenter study. Int J Oral Maxillofac Implants 1994: 9: 31-40.

[5]. Becker W, Sennerby L, Bedrossian E, Becker BE, Lucchini JP. Implant stability measurements for implants placed at the time of extraction: a cohort, prospective clinical trial. J Periodontol 2005: 76: 391-397.

[6]. Immediate placement of endosseous implants into the extraction sockets. Ebenezer V, Balakrishnan K, Asir R, Sragunar B. J Pharm Bioallied Sci. 2015 Apr; 7(1): S234-S237

[7]. Lazzara RJ. Immediate implant placement into extraction sites: Surgical and restorative advantages. Int J Periodon- tics Restorative Dent 1989;9:332-343.

[8]. Quirynen M, Van Assche N, Botticelli D, Berglundh T. How does the timing of implant placement to extraction affect outcome? Int J Oral Maxillofac Implants 2007;22:203-223.

[9]. Schropp L, Isidor F. Timing of implant placement relative to tooth extraction. J Oral Rehabil 2008;35(1):33-43.

[10]. Becker W, Goldstein M. Immediate implant placement: Treatment planning and surgical steps for successful out- come. Periodontol 2000 2008:47:79-89.

[11]. Chen ST, Buser D. Clinical and esthetic outcomes of implants placed in postextraction sites. Int J Oral Maxillofac Implants 2009;24:186-217.

[12]. Atieh MA, Payne AG, Duncan WJ, de Silva RK, Cullinan MP. Immediate placement or immediate restoration/loading of single implants for molar tooth replacement: A systematic review and meta-analysis. Int J Oral Maxillofac Implants 2010;25:401-415.

[13]. Davarpanah M, Szmukler-Moncler S. Unconventional implant treatment: I. Implant placement in contact with ankylosed root fragments. A series of case reports. Clin Oral Implants Res 2009;20: 851-856.

[14]. Lang NP, Pun L, Lau KY, Li KY, Wong MC. A systematic review on survival and success rates of implants placed immediately into fresh extraction sockets after at least 1 year. Clin Oral Implants Res 2012;23(5):9-66.

[15]. Rebele SF, Zuhr O, Hurzeler MB. Pre-extractive Interradicular Implant Bed Preparation: Case Presentations of a Novel Approach to Immediate Implant Placement at Multirooted Molar Sites. Int J Periodontics Restorative Dent. 2013 :33(1):89-96.

$\mathbf{E}$

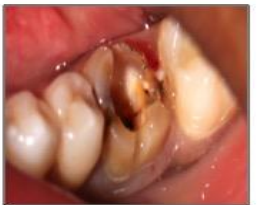

Fig.1

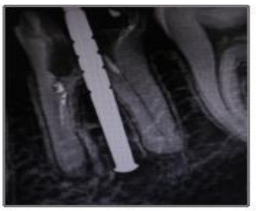

Fig.4 - measuring gauge at site to beprepared

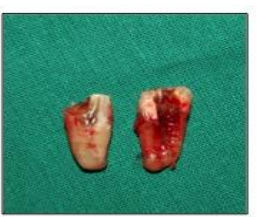

Fig.7- root fragments

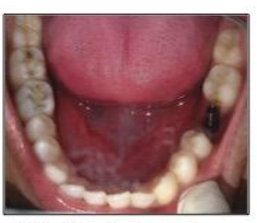

Fig.10 abutment placement

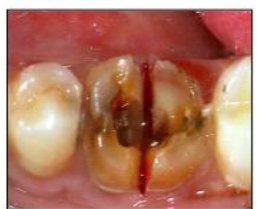

Fig.2-sectioning the tooth

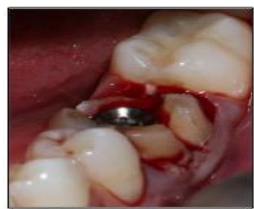

Fig.5 placement of implant

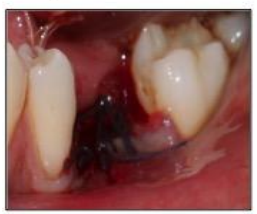

Fig.8- site is sutured

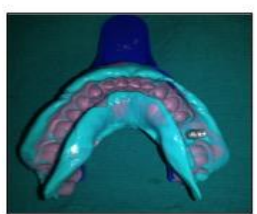

Fig.11-closed tray impression

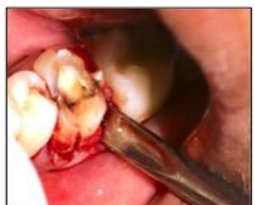

Fig.3-luxate the roots

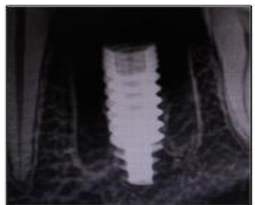

Fig.6-implant placed in interradicular septum

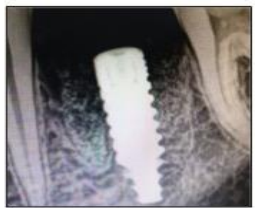

Fig.9-3 months post surgery

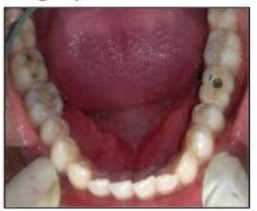

Fig.12-final prosthesis cementation 